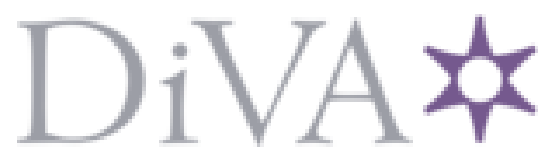

http://www.diva-portal.org

\title{
Preprint
}

This is the submitted version of a paper presented at IEEE PowerTech Conference Eindhoven, 20150702.

Citation for the original published paper:

Singh, R S., Hooshyar, H., Vanfretti, L. (2015)

Assessment of Time Synchronization Requirements for Phasor Measurement Units.

In: IEEE conference proceedings

http://dx.doi.org/10.1109/PTC.2015.7232728

N.B. When citing this work, cite the original published paper.

Permanent link to this version:

http://urn.kb.se/resolve?urn=urn:nbn:se:kth:diva-175859 


\section{Assessment of Time Synchronization Requirements for Phasor Measurement Units}

\author{
Ravi Shankar Singh \\ Aalto University \\ Espoo, Finland \\ Email: ravi.singh@aalto.fi
}

\author{
Hossein Hooshyar \\ KTH Royal Institute of Technology \\ Stockholm, Sweden \\ Email: hossein.hooshyar@ee.kth.se
}

\author{
Luigi Vanfretti \\ KTH Royal Institute of Technology \\ Stockholm, Sweden \\ Statnett SF, Oslo \\ Oslo, Norway \\ Email: luigi.vanfretti@ee.kth.se
}

\begin{abstract}
This paper assesses time synchronization sources suitable for Phasor Measurement Unit (PMU) applications in power systems. The paper investigates PMU phase error due to PMU timing inaccuracy and the presence of current and voltage transformers. This paper also shows that in practical on-field applications of commercial PMUs, the required accuracy of the time source should be better than the $31.8 \mu \mathrm{s}$ limit mentioned in the IEEE Standard for Synchrophasor Measurements for Power Systems C37.118.1-2011 and could be well in the sub-microsecond range. The laboratory test set-up is discussed in brief.
\end{abstract}

Index Terms-Phasor Measurement Unit (PMU), Time Synchronization, Total Vector Error (TVE).

\section{INTRODUCTION}

\section{A. Motivation}

Today's modern power grid is a large complex and interconnected system. An event occurring at one part of the grid may affect the operation of the whole system. Understanding these events better, requires a way to compare the power systems response at the same point of time at different locations of the grid [1]. For this purpose, PMUs are being used to provide synchronized phasor measurements to feed wide-area monitoring, control, and protection applications [2].

A common and accurate timing with reference to the Coordinated Universal Time (UTC) is required by the PMUs for correct operation and required performance level [3]. Any inaccuracy in a PMU's timing adversely affects the PMU estimation of phasor angles of the measured quantity, which in turn affects the Total Vector Error (TVE) of the phasor estimate. The TVE is the difference between the theoretical phasor value of the signal and the phasor estimate of the signal generated by a PMU. As stated in the IEEE C37.118.1-2011 standard for Synchrophasors Measurements for Power Systems [4], the time source should be accurate enough to keep the TVE within $1 \%$.

\section{B. Motivation}

Previously, work has been carried out to assess the TVE compliance criteria of PMUs in steady state conditions [5].

This work is supported by the FP7 IDE4L project funded by the European Commission, the STandUp for Energy Collaboration Initiative and by Statnett $\mathrm{SF}$, the Norwegian TSO).
These tests were performed using stand-alone relay test kits injecting the voltage and current signals directly to the PMUs. But in practical applications, PMUs receive signals via instrumentation channels consisting of current and voltage transformers (CTs and VTs) situated in the field and the control cable connecting the CTs and VTs to the PMUs. These signals after passing through CTs and VTs get phase shifted to some extent and these phase shift errors will reflect upon phasor measurements computed by PMUs [6]. As a consequence, these phase errors will also affect the accuracy requirements of PMU timing. This paper presents results from controlled experiments that show how PMU timing errors lead to phase errors, which have an impact in defining future PMU accuracy requirements (i.e. the PMU's phasor output itself, the instrumentation channel and timing sources).

The remainder of the paper is organized as follows: Section II details the available time distribution and synchronization methods for Intelligent Electronic Devices (IEDs). In section III, the timing requirements for PMUs and suitable options are discussed. Section IV assesses timing requirements of PMUs in a practical controlled experimental environment by means of real-time hardware-in-the-loop (HIL) tests. Conclusions are drawn in section $\mathrm{V}$.

\section{Time Synchronization Methods}

The need of time synchronization has increased with modernization of IEDs. Over time, many methods providing accurately synchronized time to IEDs have emerged. The most common among the other present techniques are the Global Positioning System (GPS) as a time source and the Network Time Protocol (NTP) as a method for time distribution [7]. Some other time distribution methods include standard radio transmission of time from ground based radio stations like WWVB (US) and DCF77 (Germany), Inter-Range Instrumentation Group (IRIG) B coded time signals and IEEE 1588 Precision Time Protocol (PTP) [8]. A brief overview of these methods is discussed below:

- GPS Time Source: The accuracy of GPS is in the range of $\pm 10 \mathrm{~ns}$ to $\pm 100 \mathrm{~ns}$ [7]. Its sources of error include atmospheric delays (which cannot be compensated). Factors like antenna positioning and availability of full horizon view may also affect the accuracy [7]. But even with 
all its drawbacks, the accuracy of the GPS makes it the most popular choice for high accuracy time distribution to IEDs [7].

- NTP Time Distribution: The NTP network is made up of time servers at different hierarchical levels called stratum. The topmost server is directly connected to a high accuracy reference source like an atomic clock [7]. The accuracy of NTP depends on the network topology, operating system delays and the medium of communication and is in the range of milliseconds [8].

- Standard Radio Distribution: In standard radio distribution of time the receiver device periodically synchronizes its clock to the real atomic clock whose time is transmitted using radio waves. Propagation delay of the radio waves affects the time accuracy and can cause a delay up to $30 \mathrm{~ms}$ [8].

- IRIG-B Distribution: IRIG-B is a standardized set of time-code formats and has become a popular format for time distribution to IEDs. IRIG-B provides time once per second containing information from second of the minute to the day of the year in a binary-coded decimal (BCD) format. Assisted by a GPS clock as its source, IRIG-B can distribute time with an accuracy of $\pm 500 \mathrm{~ns}$. IRIG-B is used from short to medium distance applications [8].

- PTP Time Distribution: IEEE 1588 PTP is capable of achieving accuracy levels up to $\pm 500 \mathrm{~ns}$ with the support of dedicated hardware for precision time stamping [8]. Implementation of PTP based time distribution requires the network infrastructure (like all Ethernet switches) to have hardware support for high-precision time stamping. Dedicated hardware support to implement IEEE 1588 in relays and other substation IEDs still remains limited [8].

\section{PMU TIME SYNCHRONIZATION}

The accuracy of a phasor estimate from a PMU is measured in terms of TVE. The TVE for an operational PMU under steady-state conditions should not exceed the 1\% mark [4]. Assuming no magnitude errors in the synchrophasor estimate, then a $1 \%$ TVE corresponds to a phase angle error of $0.573^{\circ}$. In time, this is about $31.8 \mu \mathrm{s}$ at system frequency of $50 \mathrm{~Hz}$ $(26 \mu s$ at $60 \mathrm{~Hz})$. The time source for PMUs should be accurate enough to keep the TVE below 1\%. It means PMU's timing errors must be less than $31.8 \mu \mathrm{s}$ in a $50 \mathrm{~Hz}$ system. Looking at the timing accuracy levels associated with the timing solutions above discussed, it can be stated, that as for now, only GPS, IRIG-B and IEEE 1588 PTP are suitable for synchrophasor applications. Hardware support for PTP in the substation IEDs and Ethernet switches in the network still remains a bottleneck in the implementation of PTP based timing solutions in substations. IRIG-B is a suitable high precision timing distribution solution and along with a GPS source, could be used effectively to distribute time to PMUs in the sub-microsecond level accuracy.

The TVE is composed of both phase errors and magnitude errors. PMU timing accuracy will directly reflect in the phase errors of the PMU and consequently, the required 1\% TVE accuracy puts a cap on timing errors. Phase errors in PMU phasor estimates are comprised by various components, including, but not limited to : time source error, delay in PMU's signal processing unit (which includes analog to digital converters and step down transformers), etc. [1].

In substations, a group of devices (collectively referred as the instrumentation channel) feed a scaled replica of the high power current and voltage signals to the PMUs as shown in Fig. 1. In practical conditions this instrumentation channel also degrades the original signal in terms of phase and magnitude. A visual example of phase error imposed by the CTs and VTs to current and voltage signals is shown in Fig. 2. These errors will affect the TVE of the synchrophasor estimate. To illustrate in simple terms, if the part of the TVE caused by the instrumentation channel could be termed as $\left(T V E_{I C}\right)$ and if the TVE caused solely due to the PMU's signal processing unit could be termed as $T V E_{P M U}$, then the total TVE (TVE $E_{\text {Total }}$ ) of the final phasor estimate by the PMU would be the sum of

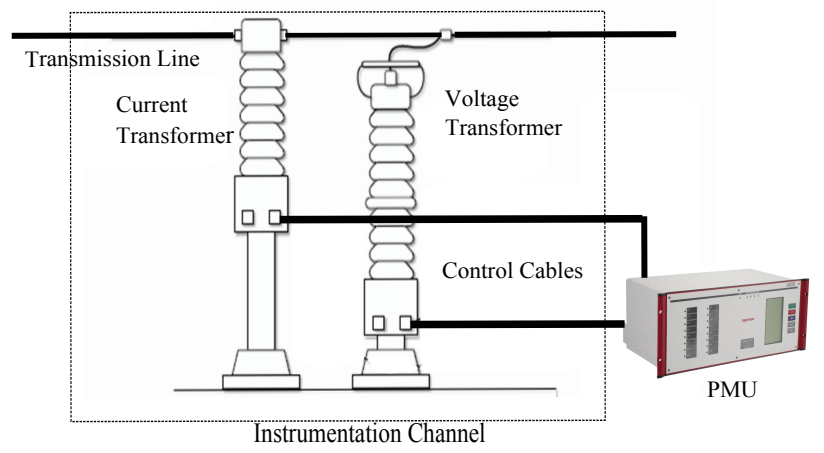

Fig. 1: PMU and Instrumentation Channel
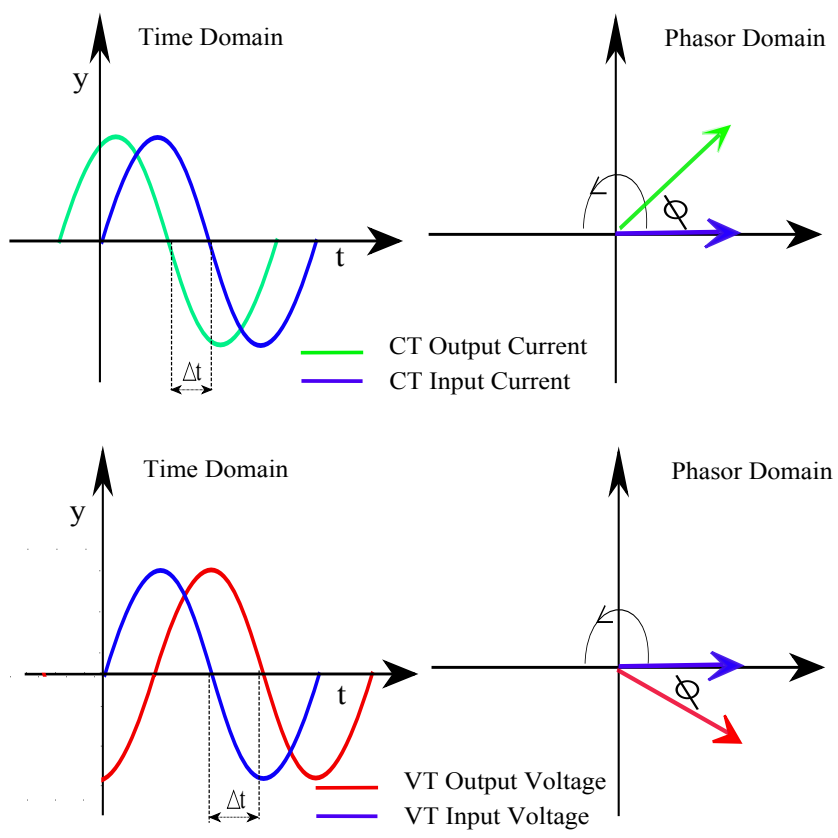

Fig. 2: Phsors representation of CT and VT outputs 
the $T V E_{I C}$ and $T V E_{P M U}$.

Testing and analysing a PMU in the lab using standalone relay test sets allows us to determine the phase error caused by the PMU's signal processing unit. However, this is not identical to the practical application when the PMU is being fed via the instrumentation channel. Phase angle errors for VTs are in the range of $\pm 4^{\circ}$ and for CTs are in the range of $\pm 2^{\circ}$ [9]. In high accuracy current and voltage transformers, the phase angle error varies between $\pm 0.1^{\circ}$. These errors increase significantly during transients especially for Capacitive Voltage Transformers (CVTs) as characteristic parameters of the components deteriorate for frequencies other than the fundamental [9]. Control cables used for connecting the instrument transformers to the PMUs and relays along with isolating switches and various non-linear burdens also impact the over all accuracy of the instrument channel. As depicted in Fig. 2, the output voltage of VT lags the original voltage waveform and the phase error is taken to be positive. Similarly, the CT output current leads the actual current signal and the phase error is assumed to be negative [10]. These phase angle errors incurred in the instrumentation channels reduce the margin for timing errors because the contribution of both the errors should not exceed the 1\% TVE limit. Therefore, the margin of error for PMU timing could be reduced significantly less than the 31.8 4 s limit specified in IEEE C37.118.1-2011. The following section presents the tests and the results to support this statement.

\section{Assessment of PMU Time ReQuirements}

In order to investigate the effect of the instrumentation channel and assess the timing accuracy required by PMUs, three test scenarios were performed on two commercial PMUs. The first test allows to identify the phase angle errors caused only by the PMU signal processing unit itself. The second test emulates an instrumentation channel that feeds current and voltage signals to the PMU, thus allowing to determine phase angle errors introduced by the combination of PMU and the instrumentation channel. In the third test, the accuracy of the timing signal synchronizing the PMU under test was was varied in real time to investigate the TVE compliance of the PMU in presence of instrumentation channel and varying timing accuracies.

\section{A. Test case 1: Stand-alone PMU}

In this test case, the phase error of PMUs due to their signal processing unit was measured. The Freja-300 relay tester kit was used to inject current and voltage signals. Balanced three phase voltage signals were injected into the PMU terminals. PMU Time synchronization was provided by distributing high accuracy IRIG-B signals originating form a GPS based substation clock. Synchrophasor data generated by the PMUs was sent to Phasor Data Concentrator (PDC) over Ethernet. Saved data on a PDC was then taken for further analysis to determine the phase error using MATLAB.

Figs. 3 and 4 show the phase angle errors for the voltage phasors calculated by two PMUs. As shown in the figures,

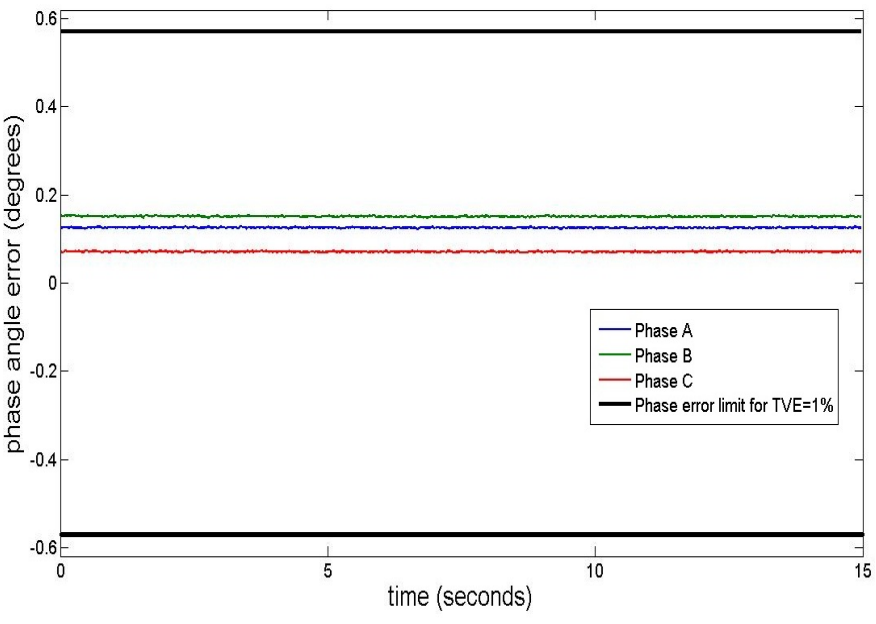

Fig. 3: Stand-alone PMU1 voltage phase error

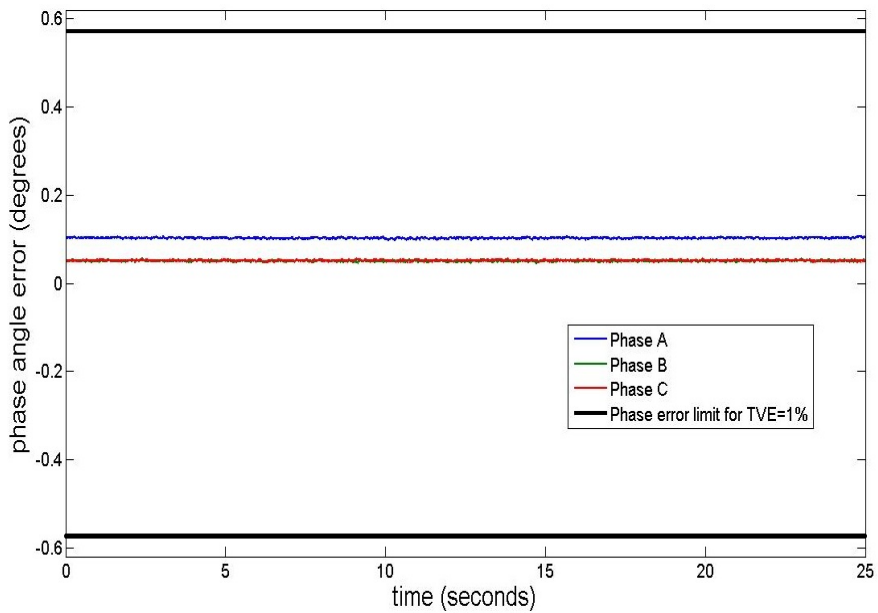

Fig. 4: Stand-alone PMU2 voltage phase error

phase angle errors for PMU1 and PMU2 were in the range of $\left[0.07^{\circ}-0.1247^{\circ}\right]$ and $\left[0.05^{\circ}-0.1^{\circ}\right]$, respectively. These errors are well within the limit of $0.573^{\circ}$ for which TVE becomes $1 \%$. Both the PMUs, time synchronized by IRIG-B time signals from a GPS substation clock comply with the requirements for TVE under steady-state conditions.

\section{B. Test case 2: PMU and Amplifiers}

This test case investigates the phase angle errors for phasors calculated by PMUs receiving input signals from CTs and VTs. These CTs and VTs were emulated using single phase Megger amplifiers SMRT1 [11]. The phase angle accuracy of the amplifier's output current and voltage signals was in the range of $\pm 0.25^{\circ}$ at $50 \mathrm{~Hz}$ [11]. This accuracy rangeis suitable to be used as a substitute for high accuracy instrument transformers whose phase angle accuracy varies between $\pm 0.1^{\circ}$. Balanced three phase voltage and current signals were generated in real-time using OPAL-RT simulator and were fed to the PMUs through the Megger amplifiers. Accurate IRIG-B time signals generated by the same real-time simulator with the same time reference as the voltage and current 


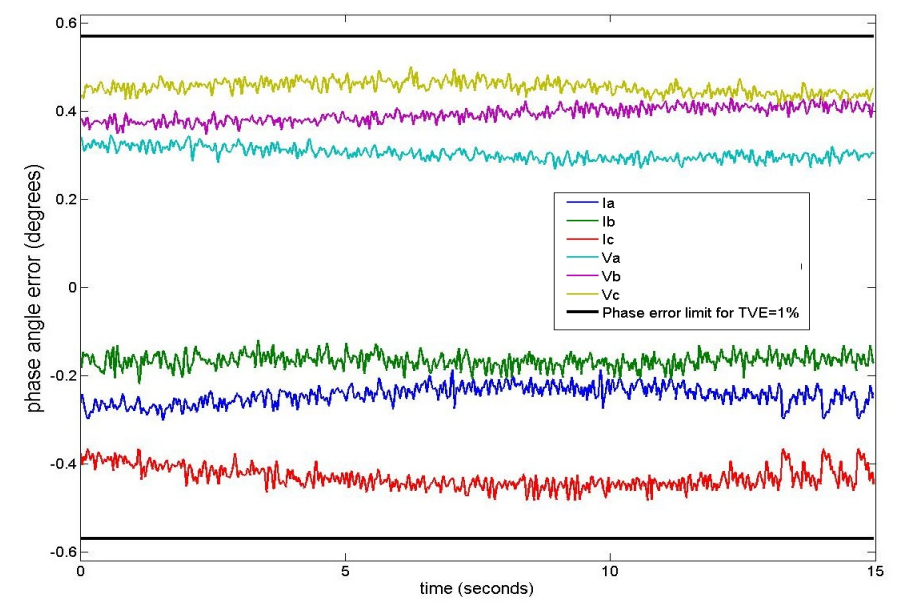

Fig. 5: PMU1 voltage and current phase errors in presence of amplifiers

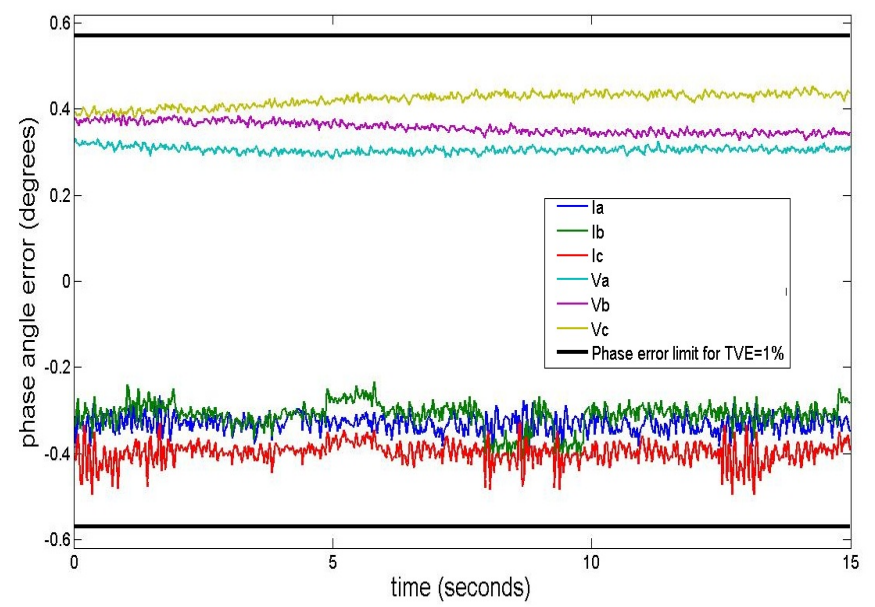

Fig. 6: PMU2 voltage and current phase errors in presence of amplifiers

signals were provided to the PMUs. Synchrophasor data were recorded in the PDC and analysed for phase error. Phase errors measured during this test are shown in Fig. 5 and Fig.6. As shown in the figures, the phase angle error for both PMUs increased. Although the time distributed was accurate up to 500ns, voltage phase angle error was observed in the range of $\left[0.332^{\circ}-0.46^{\circ}\right]$ for PMU1 and $\left[0.32^{\circ}-0.45^{\circ}\right]$ for PMU2. Phase angle errors for currents were observed in the range of negative $\left[0.187^{\circ}-0.465^{\circ}\right]$ for PMU1 and negative $\left[0.240^{\circ}-0.494^{\circ}\right]$ for PMU2. This is due to the phase angle shift caused by the CTs and VTs inside the amplifiers which are emulate the onfield CTs and VTs. The polarities of the measured current and voltage phase errors are opposite. As mentioned earlier, this is due to the fact that the voltage output from the VT lags the original voltage by certain degrees and the output current from the CT leads the original current by certain degrees. With the original phase as reference, the lagging phase error is assumed to be positive and the leading phase error is assumed to be negative. The errors are still under $0.573^{\circ}$ limit but there is very little margin left for errors due to timing inaccuracies. Depending upon the phase errors associated with CTs and VTs, the TVE of a PMU could break the limit of $1 \%$.

\section{Test case 3: PMU, Amplifiers, and Time Inaccuracy}

In this test case, to be able to vary the accuracy of PMU timing, a user-controllable IRIG-B time signal similar to test case 2 was generated in real-time using OPAL-RT simulator. Balanced three phase current and voltage signals were also generated using the real-time simulator and the same time reference. The analog current and voltage signals obtained from the simulator's output were supplied to the PMUs via an emulated instrumentation channel (current and voltage amplifiers in this case). Controlled time errors were imposed in the simulated IRIG-B signal being supplied to the PMUs. Starting with zero error, the errors were imposed in successive steps of $10 \mu \mathrm{s}$ at the points in time marked by A, B, C, D and $\mathrm{E}$ in figs. 7-13. At each step the error in timing was raised by $10 \mu \mathrm{s}$. The effect of time errors was evaluated for two different scenarios : leading i.e. advancing ahead of the correct

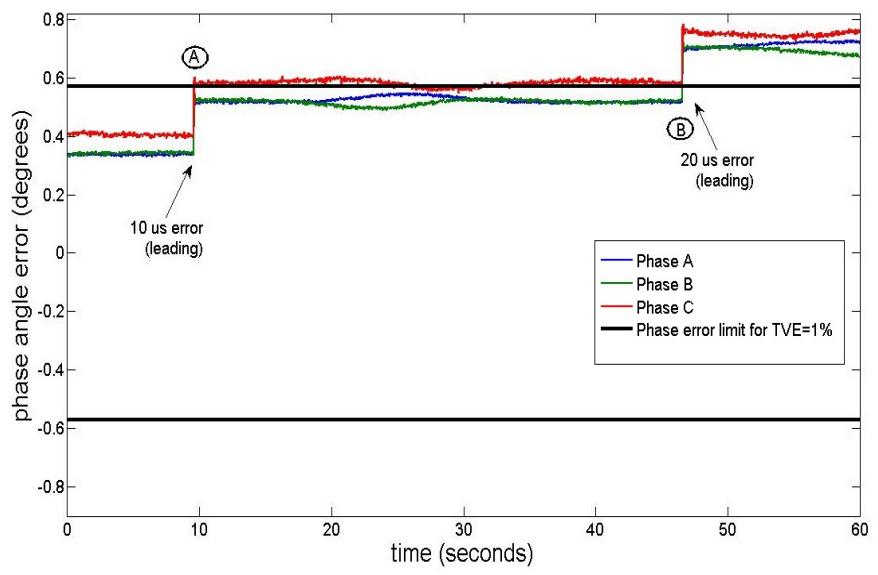

Fig. 7: PMU1 voltage phase errors in presence of amplifiers and leading time error

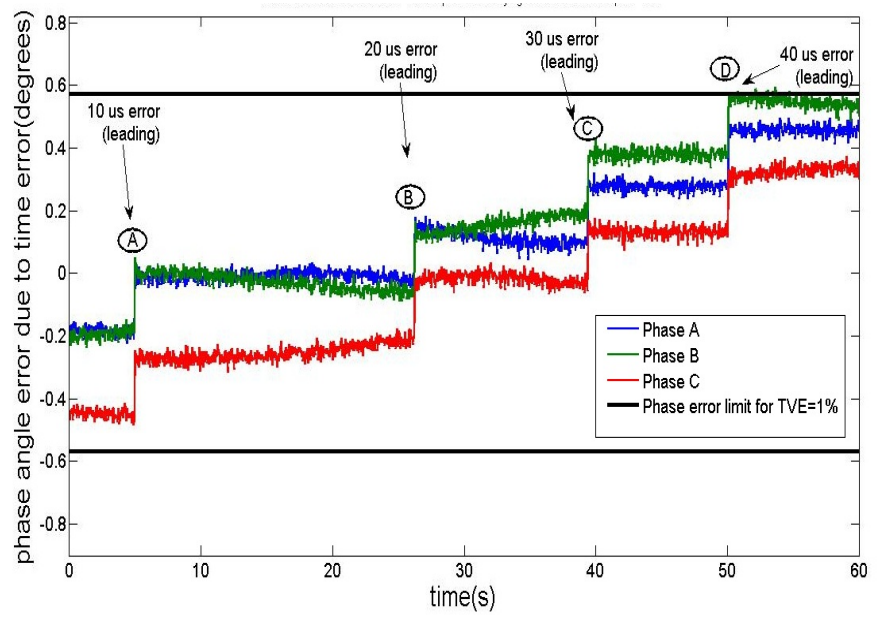

Fig. 8: PMU1 current phase errors in presence of amplifiers and leading time error 


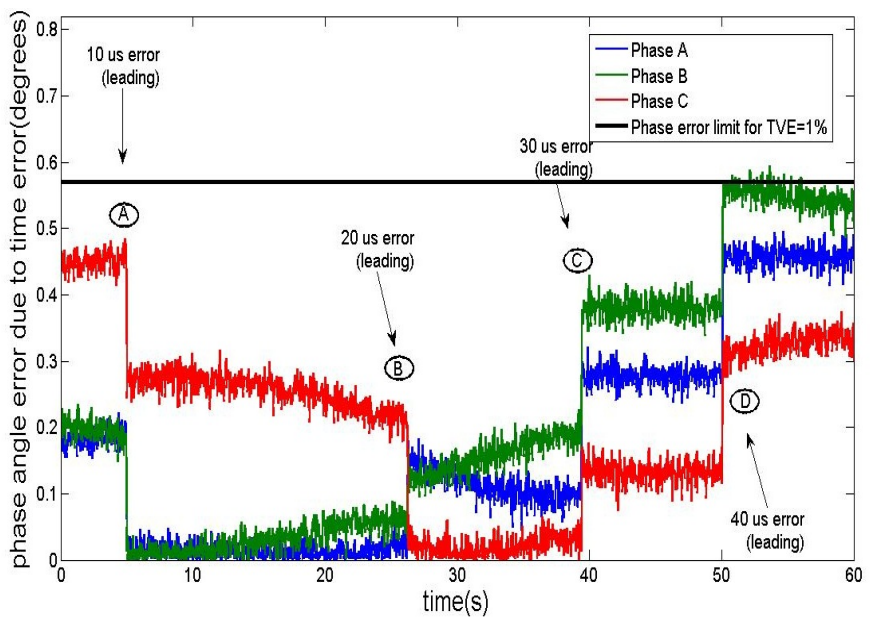

Fig. 9: PMU1 absolute current phase errors in presence of amplifiers and leading time error

time and lagging i.e. having a delay in the the time were investigated. The phase angle errors were analysed from the stored data. It should be noted that varying the time error in the IRIG-B signal, caused the PMU to give unstable and incorrect phasor estimates for a few seconds before settling down (i.e. it induced transients). Therefore, the transients in the signals have been filtered and are not included in the figures.

Figs. 7 and 8 show the effect of leading time error in steps of $10 \mu \mathrm{s}$ on the voltage and current phase errors for PMU1. As shown in Fig. 7, since the voltage signals input to the PMU were already lagging the original signals due to the phase errors introduced by the VTs, the leading time error of PMUs's clock increases this phase error in the estimated phasor. Thus, with an increased phase error due to the effect of VTs, the TVE touches the the $1 \%$ mark for one of the voltage phases only after a $10 \mu \mathrm{s}$ error in PMU timing and the TVE crosses the $1 \%$ mark for all the phases by a total timing error of $20 \mu \mathrm{s}$, which is much lower than the $31.6 \mu \mathrm{s}$

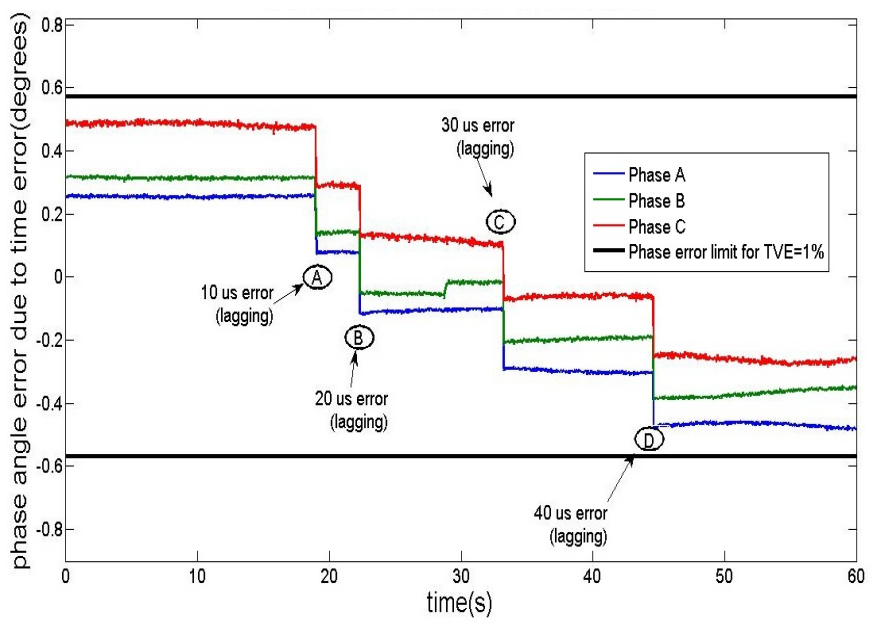

Fig. 10: PMU1 voltage phase errors due in presence of amplifiers and lagging time error limit set in [4]. The current signals show a different behaviour. As shown in Fig. 8, introducing a leading time error initially causes the measured phase error to decrease. As the timing error was further increased the measured phase error crosses zero and starts to increase in the opposite direction. This is due to the fact that the current signal input to the PMU was leading the original signal due to the effect of the phase error introduced by CTs. So when the time is erroneously advanced, up to a certain magnitude of timing error, the effect of leading timing error decreases the effect of the leading phase error and the PMU makes a better phasor estimate of the current signal. At a certain time lead error, its effect completely cancels out the effect of error in phase caused by the CTs. Afterwards, any increase in leading timing error, results in an increase in the measuresd phase error but in opposite direction. The phase angle error will start to increase again but in opposite direction.

This phenomena is also depicted in Fig. 9 where the absolute value of the phase errors were plotted. As shown, the phasor error initially decreases before increasing again.

Next, lagging timing errors were imposed on PMU1 by delaying the time in successive steps of $10 \mu \mathrm{s}$. The effects on the phase errors of voltage and current can be seen in Figs. 10 and 11. As shown in Fig. 10, as the lagging timing error increases, the voltage phase error initially decreases and then increases in the negative direction. This is due to the fact that the voltage signals that feed the PMU via VTs lag the original voltage signals, therefore when the time is delayed, the measured phase error starts decreasing first towards zero and then increasing in the negative direction. However, as shown in Fig. 11, the measured phase error of the current signal increases in the negative direction resulting in one of the phases crossing the phase error limit at a time delay error of $10 \mu s$ and with an delay error of $20 \mu s$ all the phases had crossed the 1\% TVE phase error limit. Both the time errors were much lower than the $31.6 \mu \mathrm{s}$ limit set in [4].

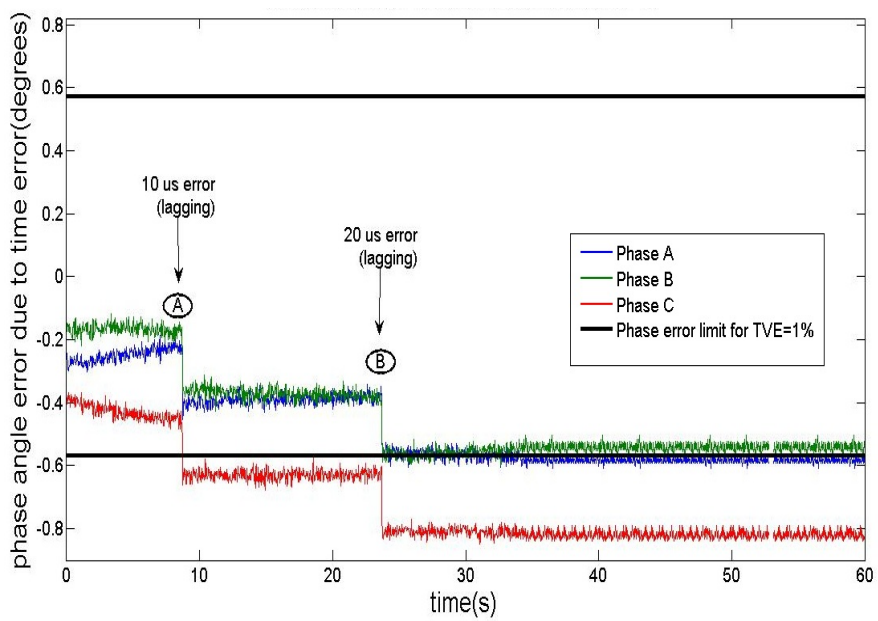

Fig. 11: PMU1 voltage phase errors in presence of amplifiers and lagging time error 


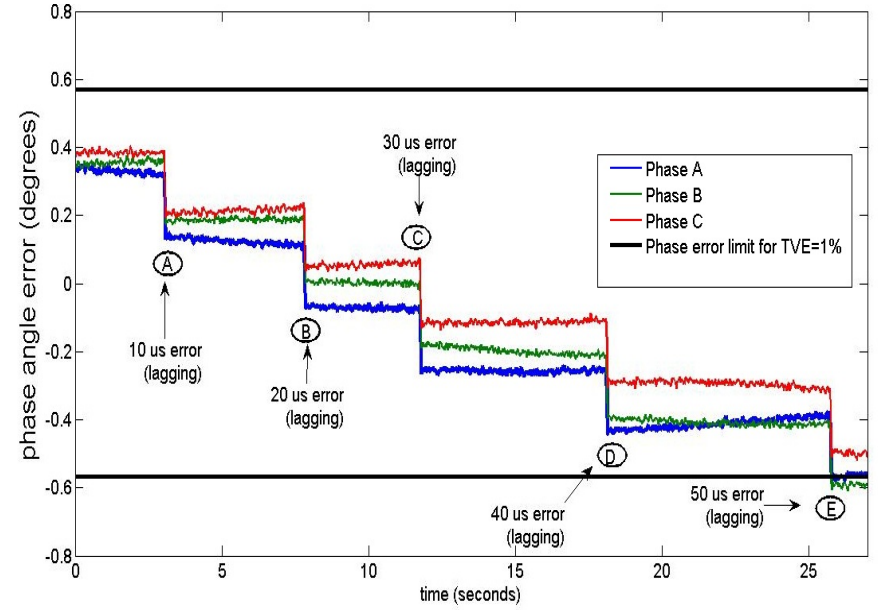

Fig. 12: PMU2 voltage phase errors in presence of amplifiers and lagging time error

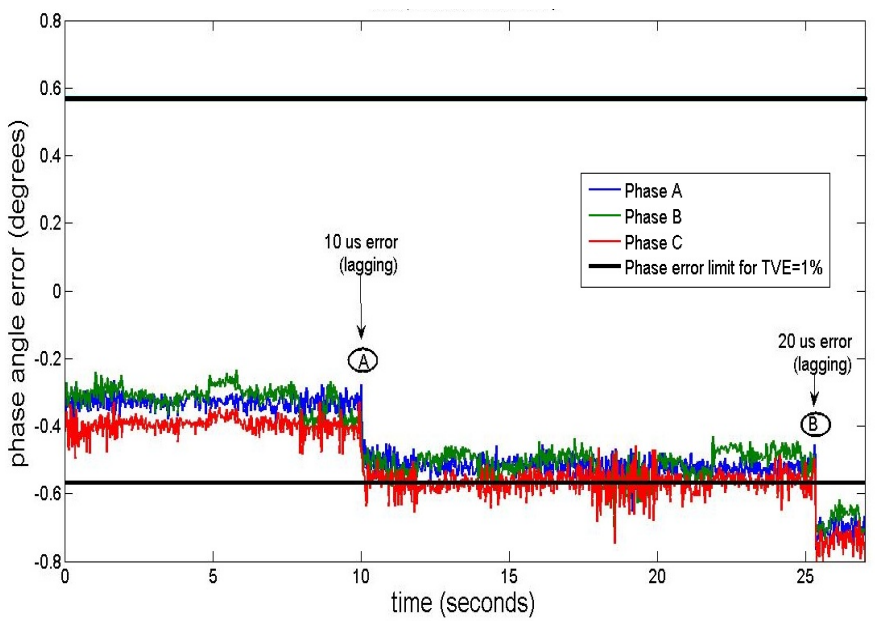

Fig. 13: PMU2 current phase errors in presence of amplifiers and lagging time error

As discussed, both of the above mentioned time error scenarios (leading and lagging), measured phase errors of either the current or the voltage signals violate the 1\% TVE rule even before the timing error reaches the $31.8 \mu \mathrm{s}$ limit, set in [4]. A similar set of tests were repeated for PMU2. Figs. 12 and 13 display the measured phase errors in the case of lagging timing errors. In this case, it can be seen that the phase error for the current signals reach the 1\% TVE mark in negative direction after $20 \mu \mathrm{s}$ error was introduced at point B. However, similarly to PMU1, the voltage phase error decreases and then increases in the opposite direction.

All the results indicate that PMUs require a time source with a better accuracy than the $31.8 \mu \mathrm{s}$ limit set in [4]. These results also confirm that in order to limit the TVE of PMUs below $1 \%$, high accuracy time synchronization methods such as GPS, IRIG-B and hardware supported PTP with submicrosecond accuracy are required. Other available methods of time distribution such as NTP and standard radio are not suitable for PMU applications.

\section{CONClusion}

The paper discussed different sources and distribution methods to provide timing solutions and compared their usability as time sources for PMU time synchronization. It was seen that GPS, hardware supported PTP and IRIG-B are capable time synchronization solutions for PMU applications. To investigate the timing requirements of commercial PMUs, two commercial PMUs were tested for their phase errors by means of realtime HIL tests using amplifiers to emulate CTs and VTs of the instrumentation channel. It was shown that for two different commercial PMUs the final phasor measurement error could be quite large due to its instrumentation channel. These factors of TVE errors would affect the accuracy of the PMU measurements and will also make the timing requirements more stringent. This, PMU timing accuracy requirements need to be evaluated while keeping the effects of instrumentation channel errors in consideration. The tests showed that even a time source error of $10 \mu \mathrm{s}$ could be sufficient to make TVE higher than $1 \%$. It has been concluded that to leave some margin for other errors, the required timing source accuracy could be well in sub-microsecond range.

\section{REFERENCES}

[1] B. Dickerson, "Precision Timing in the Power Industry: How and Why We Use it," [Online]. Available: http://www.arbiter.com/news/ technology.php?id=4.

[2] A. Bose, "Smart Transmission Grid Applications and Their Supporting Infrastructure" IEEE Trans. Smart Grid, vol. 1, pp. 11 -19, 2010.

[3] IEEE Guide for Synchronization, Calibration, Testing, and Installation of Phasor Measurement Units (PMUs) for Power System Protection and Control, C37.242-2013, March 2013.

[4] IEEE Standard for Synchrophasor Measurements for Power Systems, C37.118.1-2011, Dec 2011.

[5] M. S. Almas, J. Kilter and L. Vanfretti, "Experiences with steady-state PMU compliance testing using standard relay testing equipment", in Proc. Electric Power Quality and Supply Reliability Conference (PQ), 2014, pp. 103-110.

[6] Z. Wu, K. Thomas, R. Sun, V. A. Centeno and A. G. Phadke, "Threephase instrument transformer calibration with synchronized phasor measurements", in Proc. 2012 IEEE PES Innovative Smart Grid Technologies (ISGT), pp. 1-6.

[7] L. Wang, J. Fernandez, J. Burgett, R.W. Coners, "An evaluation of network time protocol for clock synchronization in wide area measurements", in 2008 IEEE Power and Energy Society General Meeting Conversion and Delivery of Electrical Energy in the 21st Century, pp. $1-5$.

[8] Jackie Peer, Eric Sagen, Shankar Achanta and Veselin Skendzic, 'The Future of Time: Evolving requirements for Precize Time Synchronization in the Electric Power Industry", Schweitzer Engineering Laboratories, Inc., 2011, [Online]. Available: https://www.selinc.com/WorkArea/ DownloadAsset.aspx?id=9995.

[9] Z.Huang; B. Kasztenny; V. Madani; K. Martin et al, "Performance evaluation of phasor measurement systems", in Proc. Power and Energy Society General Meeting - Conversion and Delivery of Electrical Energy in the 21st Century,2008, pp. 1-7.

[10] Walter A. Elmore, Protective Relaying: Theory and Applications, CRC press, 2003, p. 82,92.

[11] Megger, SMRT1, Single Phase Relay Tester, [Online]. Available: http: //www.megger.com/us/products/ProductDetails.php?ID=1529 